\title{
Control Problems in Armored Face Conveyors for Longwall Mines
}

\author{
Alan R. Broadfoot, Member, IEEE, and Robert E. Betz, Member, IEEE
}

\begin{abstract}
This paper is a tutorial discussion of the current difficulties being experienced with the performance of armored face conveyor (AFC) drive systems, as used in longwall mining. It presents the traditional approaches to the design of the drive system and highlights the inadequacies. The final part of the paper presents a possible solution approach using variable-speed drive systems, emphasizing the advantages of this approach. The paper is significant, in that it discusses, in one document, a number of problems related to the operation of longwall AFC's. Furthermore, it presents a solution path for these problems. The details of the control strategies to solve the problems highlighted are left to a companion paper [4].
\end{abstract}

Index Terms - Armored face conveyor, control problems, inverters.

\section{INTRODUCTION}

A RMORED FACE conveyors (AFC's) used in longwall mining applications are a complicated electri$\mathrm{cal} /$ mechanical system. A typical drive train for an AFC consists of an induction motor connected to a fluid coupling, which, in turn, is connected to a reduction gearbox. The output shaft of the gearbox is then connected to a drive sprocket. In its crudest form, the AFC can be seen to be two electric motors connected to either end of a circular chain (or chains) which run through steel chain guides. The purpose of the electric motors is to pull the chain and its associated cross bars along a flat steel pan running between the motors and, consequently, transport any material sitting on the pan with it. In the longwall application of these conveyors, the material being carried is coal. As one can well imagine, the friction forces associated with such a conveying system are very large, and the electrical drive machines have to produce substantial torques. The calculation of the friction forces which allow the sizing of the motors and evaluation of mechanical forces are presented in [1] and [2].

This deceptively simple system for conveying material along a longwall coal face, however, has a number of problems. Almost all these problems are due to an interplay

Paper PID 97-26, presented at the 1996 Industry Applications Society Annual Meeting, San Diego, CA, October 6-10, and approved for publication in the IEEE TRANSACTIONS ON INDUSTRY APPLICATIONS by the Mining Industry Committee of the IEEE Industry Applications Society. Manuscript released for publication October 13, 1997.

A. R. Broadfoot is with AMP Control SWG Pty. Ltd., Newcastle, NSW 2322, Australia.

R. E. Betz is with the Institute of Energy Technology, Department of Electrical Energy Conservation, Aalborg University, DK-9220 Aalborg East, Denmark, on leave from the Department of Electrical and Computer Engineering, University of Newcastle, NSW 2308, Australia.

Publisher Item Identifier S 0093-9994(98)02564-X. between the electrical and mechanical parts of the system. The drive train configuration mentioned above is commonly replicated at both the maingate and tailgate ends of the face. This is not necessarily the only configuration, for example, one can have a single-drive AFC. However, for longer AFC's, this is not common, as the motor has to be larger and, in the confined space, of the face this can be a problem. Furthermore, single-motor solutions result in larger peak tensions in the chains. The lower weight at the AFC ends in a dual-motor configuration also has the advantage that the AFC is easier to advance with reduced-size hydraulics on the chocks. However, these benefits are, to some degree, offset with some problems, as will be seen later.

The problems associated with the operation of the AFC can be broken down into two main categories, electrically related problems and mechanically related problems.

\section{EleCtrical PRoblems IN AFC Systems}

The problems associated with the electrical side of the AFC system can be itemized as follows:

1) voltage dip due to the high starting currents;

2) possible stalling due to externally imposed voltage fluctuations;

3) a difference in the voltage supplied to the two motors in a dual-drive system;

4) variation of the motor characteristics;

5) possible large transient torque pulsations in the machines.

Let us now consider each of these in some detail.

\section{A. Voltage Dip}

The supply to an AFC is usually very weak, due to the distance from the surface distribution point to the main underground transformer and the distance from this transformer to the AFC drive motors. As mentioned in Section I, the motors used to drive an AFC are usually squirrel-cage induction motors. These machines usually employ a deep bar rotor or a double-cage rotor, in order to generate a reasonable value of starting torque. Nevertheless, such machines still have the induction machine characteristic of large overcurrent when started direct on line (DOL) at mains frequency and voltage. These large currents result in substantial voltage drops at the terminals of the AFC motors, due to the effect of the supply line impedances.

The voltage dip at the terminals has a significant effect on the starting torque of the AFC motors. This can easily be seen 
from the approximate expression for the steady-state torque of a single-cage three-phase induction machine [5]:

$$
T_{e} \approx \frac{3 p_{p} s V^{2} R_{2}^{\prime}}{\omega\left[\left(R_{2}^{\prime}\right)^{2}+\left[s\left(X_{2}^{\prime}+X_{1}\right)\right]^{2}\right]}
$$

where

$$
\begin{aligned}
& p_{p} \triangleq \text { pole pairs; } \\
& s \triangleq \text { rotor slip; } \\
& R_{2}^{\prime} \triangleq \text { referred rotor resistance; } \\
& X_{2}^{\prime} \triangleq \text { referred rotor leakage impedance at } 50 \mathrm{~Hz} \\
& X_{1} \triangleq \text { stator leakage impedance at } 50 \mathrm{~Hz} \\
& \omega \triangleq \text { applied frequency rad/s. }
\end{aligned}
$$

This fundamental expression holds for each of the two cages in a double-cage induction machine. Notice that, for a given value of slip, the torque is related to the square of the incoming supply voltage $V$. This high sensitivity to voltage means that even modest drops in the terminal voltage can have quite a dramatic effect on the electromagnetic torque produced by the machine.

The other source of voltage dip is not due to the motor's self current, but due to other disturbances on the supply system. For example, disturbances can be caused by a supply authority, a distribution fault, or the starting of a large drive somewhere else on the system. The most common source of disturbances is a fault on the underground distribution system. Such faults may be phase-to-earth faults, phase-to-phase, two-phase-to-earth, or a bolted three-phase fault. The worst type of fault is the latter.

When a fault occurs, there is a certain time before the circuit breakers clear the fault. This time is critical in terms of being able to restart an induction motor. This can be seen in Fig. 1. This diagram shows a typical steady-state torque-slip characteristic for an induction machine at different voltage levels. If there is a drop in voltage, then (neglecting the transients) there is a movement from curve 1 to curve 3 . If one assumes that the load torque requirements stay at the same level, then, when the voltage drops, the machine will decelerate, the rate of deceleration being determined by the difference between the torque being produced by the machine and the load torque. This deceleration results in a change in the slip of the machine. Depending on the magnitude of the voltage dip and the time before the fault is cleared, the machine may either regain its original speed or it may stall.

If the voltage has dropped so that the machine is operating on curve 3 in Fig. 1, and the machine was originally operating at a slip of $s_{o}$, then the torque being produced is $T_{v d}$, which is clearly much less that the required load torque $T_{L}$. Consequently, the machine will slow. This, in turn, implies that the slip of the machine will increase. If the time that elapses before the fault is cleared is such that the slip increases to $s_{u}$, then even if the supply is restored to normal voltage (i.e., the machine now operates on curve 1), the load torque intersections the machine torque-slip curve at an unstable point (i.e., all slips greater than $s_{u}$ ), and the speed will continue to drop until the machine stalls.

Remark 1: The above secenario, while illustrative, is not accurate, since the current to the machine will increase considerably in the high slip operation region. This, in turn, means

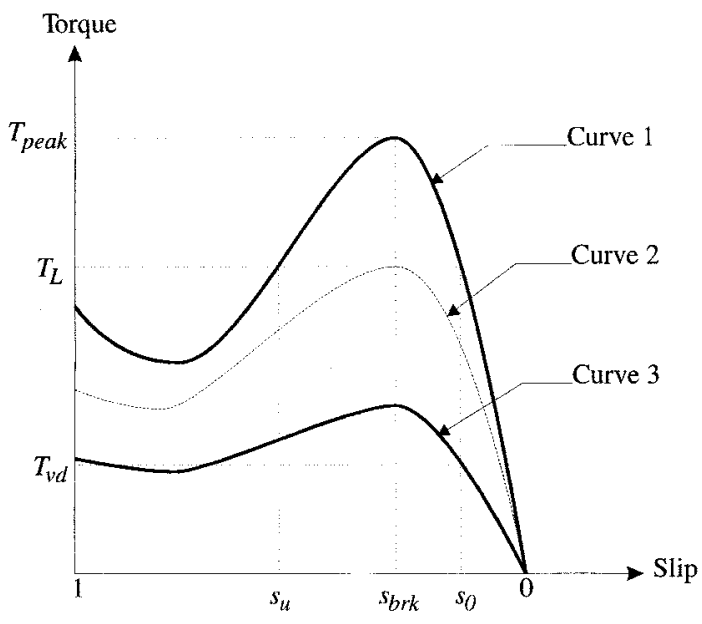

Fig. 1. Torque-speed characteristic for an induction motor with varying voltage.

that the voltage will not return to the normal value, due to the cable impedance drop. This effect would tend to make the actual fault clearance time less than in the case above. However, the load torque in the example is very much a worst case, since the load torque will drop considerably with AFC speed (the load is predominantly friction based). This effect would tend to make the falult clearance time longer than in the case above. How these two effects balance would depend on the specific situation.

To summarize, the main consequence of voltage dip on the AFC system performance is that the drive motors may stall during start or after a supply disturbance. It is unfortunate that high torque requirements usually occur at the initial start of the AFC (in order to overcome the static friction of the system), precisely where the steady-state torque can be substantially lowered by voltage drop. This can become a major problem when attempting to start a fully loaded or overloaded AFC. In the latter situation, the last resort is to use men to shovel the coal off the AFC to lighten the load. Clearly, such an eventuality would cost substantial production time and, therefore, money.

Remark 2: It should be noted that fluid couplings are used to help minimize the startup voltage drop problem by progressively applying load to the machine as the machine shaft speed increases. The large initial current (and, hence, voltage drop) still occurs, but, by the time the fluid coupling transmits torque to the AFC, the motor currents are substantially lower.

\section{B. Motor Voltage Differences}

The physical configuration of longwall faces means that the distance of the maingate and tailgate motors from the underground distribution transformer are substantially different. Consequently, the voltage drops experienced due to line impedances are different. This results in the following adverse effects on the performance of the AFC system.

1) The starting torques of the two drive motors are different, and the motor with the lower voltage is not contributing its full complement of torque. As a result, a fully loaded AFC may not start. 
2) The torques under steady state may not be balanced. The degree of imbalance depends on the slip characteristics of the machines. For example, if the torque slip curve is very steep and there is a considerable load on the machines, then a voltage difference between the machines can result in a substantial difference in the torque being produced by each motor. This can result in one of the motors and its associated drive train being overloaded and, consequently, its life being shortened. In addition, the ability of the AFC system to remain operating if there is a sudden increase in the load (as happens if a section of the face delaminates and falls onto the AFC) is compromised.

It was shown in [4] that the total starting torque is influenced greatly by the voltage difference between the two machines. If the system is not heavily loaded, however, the torque difference may be reasonably small during steady state, although this is dependent (as mentioned previously) on the torque slip characteristic of the motors.

The second effect is very important from a system reliability viewpoint. An AFC may be working satisfactorily from a starting perspective, but the mismatch in the drives can result is premature failure of equipment, with the corresponding loss of production and capital repair costs.

\section{Variation of Motor Parameters}

The consequences of motor parameter variations are very similar to the effects cited in the previous section. The main parameter that affects the torque production is the value of the rotor resistance for the induction machine. This is also the parameter that exhibits the most variation. This variation is not necessarily due to production variations, but due to internal heating of the rotor during operation. Differential heating can occur between the tailgate and maingate motors, due to the different loads experienced, leading to different torques, even if the voltage supply to the motors is approximately the same.

To gauge the effects of rotor resistance variations, one needs to ascertain the sensitivity of the torque to the rotor resistance. The sensitivity analysis can be carried out by differentiating (1) with respect to $R_{2}^{\prime}$. This leads to a rather messy expression, from which it is hard to draw any general conclusions. However, if one plots the torque-slip characteristics for a motor with a low $R_{2}^{\prime} /\left(X_{2}^{\prime}+X_{1}\right)$ ratio (i.e., steep torque slip curve), then it can be seen for a constant slip that a very small change in the torque-slip characteristic will result in a relatively large change in the torque. This fact results from the very steep angle of the characteristic for small slip machines. It can be more formally shown that $\partial T_{e} / \partial R_{2}^{\prime} \propto 1 /\left(R_{2}^{\prime}\right)^{2}$ in these regions. For larger values of $R_{2}^{\prime} /\left(X_{2}^{\prime}+X_{1}\right)$, the $X_{2}^{\prime}+X_{1}$ terms become important in the $\partial T_{e} / \partial R_{2}^{\prime}$ expressions and, consequently, the influence of the $R_{2}^{\prime}$ terms decreases. This situation occurs in larger slip induction machines, that are more common in mining applications. These effects can be seen diagrammatically in Fig. 2. For both sets of curves in this figure, the rotor resistance is increased slightly. If the speed of the motor remains fixed, then, for the case of low rotor resistance, the change in the torque of the motor is $\delta T_{1}$ while

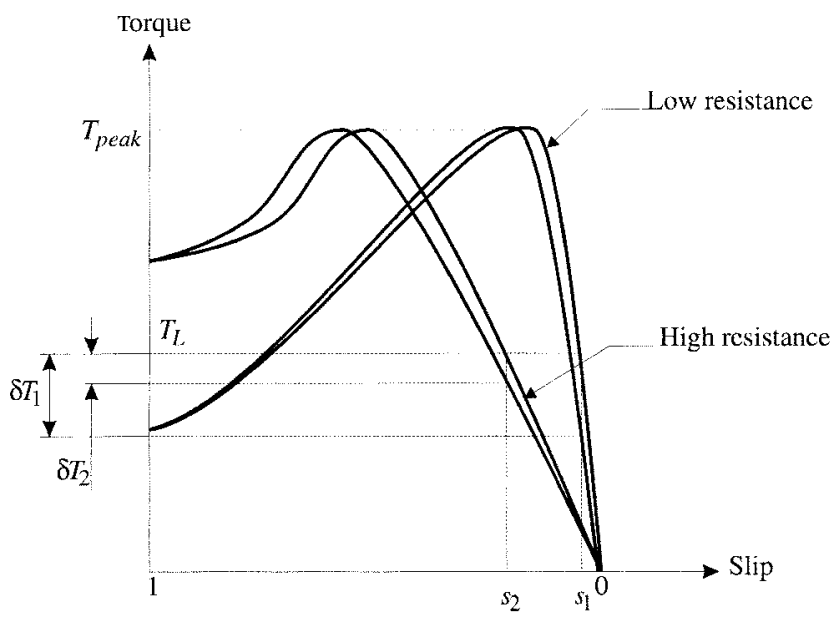

Fig. 2. Variation of torque with variation of rotor resistance for high- and low-resistance rotors.

the slip remains at $s_{1}$. For the case of high rotor resistance, the change in the torque with a change in rotor resistance is $\delta T_{2}$. It can be heuristically seen from this sketch that the low rotor resistance machine is going to be much more sensitive to a change in $R_{r}$ than the high rotor resistance machine.

The other factor that influences the sensitivity to rotor resistance changes is the voltage level. As the voltage increases, the sensitivity increases in proportion to the square of the voltage. This can be seen if one evaluates the $\partial T_{e} / \partial R_{2}^{\prime}$ term. Machines in mining applications will normally be operating at rated voltage, therefore, the sensitivity to the rotor resistance due to the voltage level will be maximized.

Remark 3: The overall conclusion is that sensitivity to changes in the rotor resistance are very dependent on the particular machine being used. Machines with a low $R_{2}^{\prime} /\left(X_{2}^{\prime}+\right.$ $X_{1}$ ) ratio will tend to be more sensitive than those with a higher ratio.

\section{Transient Torque Pulsations}

When an induction machine is started with mains frequency and voltage input, large electrical transients usually result. These cause large electromagnetic torque transients. These transients can be several times the rated torque of the machine and can oscillate positive and negative to approximately the level of the steady-state peak torque. If the motor was directly connected via a gearbox to the $\mathrm{AFC}$, these torques may be large enough to actually break the AFC chains. At the least, the life of the gearbox would be drastically shortened.

Fortunately, the fluid coupling included in most AFC drive trains alleviates such problems. The slip in the fluid coupling prevents the shaft transients during startup from being transferred directly to the gearbox and AFC. However, while the fluid coupling does this job satisfactorily, it brings with it a number of other problems (see Section III-C).

An alternative approach to address the starting problem is to use two-speed motors. These motors effectively have two pole numbers that can be changed by using a set of contactors. Using this approach, higher average starting torque can be obtained by using the high pole number, with a switch 
to the lower pole number occurring at some higher speed. However, the torque pulsation problem at startup still remains. Furthermore, there is another set of pulsations that occur when the pole changeover occurs, although these pulsations are significantly smaller than those experienced with a single-pole motor DOL start.

\section{E. Thermal Effects}

Thermal effects refer to the heat buildup in the motor during a normal start or during repeated starts over a short duration. Consider a heavily loaded AFC. If the motors are able to start the AFC, the low average starting torques can result in unacceptably long acceleration times. During this acceleration period, high currents flow into the motor, resulting in motor heating. Excessive temperatures can damage the motor insulation, resulting in, at worst, a winding insulation breakdown and, at best, a decreased lifetime for the machine.

These thermal effects can also result in a reduced start/stop cycle time for the machine. In situations where there is a large load on the AFC, many start/stop cycles can be executed in a relatively short period of time. This can result in significant temperature rises in both the motor and the fluid coupling (if present). If the process is repeated too many times, then motor or fluid coupling failure may result.

\section{Mechanical Problems in AFC Systems}

As mentioned in Section I, the mechanical problems in AFC systems are related to the electrical problems in the sense that some mechanical issues reflect on the electrical behavior and vice versa.

The main mechanical issues that are relevant to the performance of the AFC are as follows:

1) variations in the specifications of the gearboxes;

2) variations in the sprockets driving the chains;

3) variation in the performance of the fluid and wet clutch couplings.

Clearly, the overall loading determines the kilowatt rating of the motors. This is not a control issue, and it is addressed in [1] and [2].

\section{A. Gearbox Variations}

The gear ratios of so-called identical gearboxes can vary. Therefore, if the output shaft speeds of the tailgate and maingate gearboxes are the same (which is forced by the presence of the chain between them), then this implies that the input speeds are different. This, in turn, means that the maingate and tailgate motors are operating at different slips. As discussed previously, the different slips mean that the two motors will produce different torques. For example, in a typical case documented by British Coal [3], a right-angled gearbox on the maingate had a gear ratio of 28.055-1, while the tailgate gearbox was 28.47-1. Under AFC full-load conditions, the maingate drive unit was measured at approximately $30 \%$ full load, due to the difference in the slips of the two drive motors.

One obvious solution to this problem is to select the gearboxes so that the ratios are exactly matched. This may work when a system is initially installed, but it does not work when there is a breakdown and one of the gearboxes is replaced.

There are two main regions of operation for an AFC motor: 1) the acceleration phase as the AFC ramps up to speed and 2) the steady-state running condition. Gearbox mismatches have effects on both of these. During the initial acceleration phase, the torques of the machines will usually be very close, since the slip is very close to one during this time. The torque is not very sensitive to variations in slip in this region. Therefore, gearbox mismatch, unlike voltage mismatch, does not have a dramatic effect on the starting properties of the machine. However, in steady state, where the motors are operating on the negative slope section of the torque slip characteristic, there is high sensitivity to the slip frequency. Consequently, there can be a substantial steady-state torque error between the two machines.

It should also be noted that similar gearboxes can also have different efficiencies. This also results in different gearbox output torques being produced for identical input torques.

Remark 4: Gearbox mismatches have their major effect on the steady-state performance of the machine, unlike the voltage mismatch, which tends to affect the startup performance more. Therefore, gearbox mismatches tend to place long-term stress on the more heavily loaded motor and its fluid coupling.

\section{B. Sprocket Variations}

Variations in the precise physical dimensions of the sprockets can cause similar effects to those outlined above for the gearbox variations. However, due to the much simpler nature of the sprockets, the degree of variation is much smaller and can usually be neglected relative to the other effects. In addition to the errors in physical dimensions, sprockets also have an efficiency that is lower than $100 \%$. However, the loss in efficiency and the efficiency variations between sprockets are minor in relation to the other component variations and can, therefore, be neglected.

\section{Fluid Coupling Variations}

Fluid couplings, as discussed in Section I, perform a very important function in a traditional AFC drive train. However, due to the nature of the device, they introduce another level of variation on the performance of the AFC drive system. One of the most important factors that influences the performance of fluid couplings is the degree of fluid fill in the device. The slip through the coupling is directly related to this. The viscosity of the liquid also has an influence. Clearly, this will change with temperature, which again influences the slip through the device. This can be a major source of variations in the torque transmitted, since the slip (and, therefore, the power dissipated in the fluid coupling) will increase with a loss of viscosity. Therefore, there is a positive feedback effect that tends to increase the slip. This can lead to overheating of the fluid and blowing of the pressure release plugs. The heating problems in couplings are exacerbated by start/stop cycling of the drive system, since they are operating in their highest power dissipation region during these events.

The variable-fill fluid coupling allows crude compensation 
to be achieved for mismatches in other parts of the drive system (e.g., gearbox mismatch). However, the lack of precision that can be obtained and the variations that occur in the fluid coupling during the operation of the system means that this is largely ineffective.

\section{Wet Clutch Couplings}

This type of coupling is a recent mechanical solution to load-sharing and startup problems. The coupling consists of a clutch lubricated with water, the plate pressure being controlled via hydraulics. The hydraulics, in turn, are controlled via a programmable logic controller (PLC) or similar device executing a proportional/integral (PI) control algorithm to limit the current being drawn by the machine, while at the same time allowing the AFC to accelerate in a reasonable time. Additional control can then be added to this to allow better steady-state load sharing between the drives.

Wet clutch technology is relatively new on the AFC control scene. Therefore, it is difficult to make a definitive statement about the usefulness and robustness of this technology in solving the many existing AFC problems. However, a few preliminary observations and assertions can be made. Firstly, the technology seems to be a crude technique to solve the problems when compared to the modern solutions proposed in this paper. Secondly, the efficiency of the coupling would not be very good. This, in turn, leads to problems in relation to heat dissipation from the clutch. For example, current installations pass cool water through the coupling assembly, discarding the water after use. This is a very wasteful use of water resources. To overcome this, a closed circuit cooling system with a heat exchanger can be used, but this is expensive and difficult to house in the confined space near the longwall face. Thirdly, a technology that relies on friction between plates to couple power is suspect from a reliability viewpoint.

\section{SUMMARIZING}

The main effects of the above electrical and mechanical problems on the performance of the conventional AFC designs can be summarized as follows.

- Voltage drop at the AFC start due to the large currents drawn by induction machines can result in severe starting problems with conventionally designed AFC's.

- Parameter variations for gearboxes, motors, supply cables, and fluid couplings can result in large differences in the torques contributed by the maingate and tailgate motors on the AFC. This, in turn, results in starting problems, as well as undue stress on the dominant motor and its drive train, with its consequent reliability implications.

- Mechanical techniques, such as wet clutches, which are intended to overcome starting and load-sharing problems, actually generate new difficulties in the operation of the AFC.

\section{Traditional Design Techniques}

Existing AFC's clearly work and produce large volumes of coal despite all the problems cited previously. This is achieved by being very conservative in the design of the system components. For example, British Coal recommendations for the selection of the AFC drive motors were as follows.

1) Use a design factor of 0.8 in the selection of the motors to allow for mismatched tolerances, i.e.,

$$
\mathrm{kW}(\text { required })=\frac{\mathrm{kW}(\text { designed })}{0.8}
$$

2) The required kilowatts should then be rounded up to the next motor size to give a further safety margin.

Clearly, these design rules are very crude, but they have been found to be a reasonable compromise between excessive capital expenditure and system reliability. The rules have been derived heuristically using basic rules of thumb. The mismatches are never actually measured for the components, but, from experience, the 0.8 factor was chosen to embody all these uncertainties. The result is a potentially very much overdesigned motor system. This overdesign can actually contribute to the voltage dip problems by increasing the startup currents. Furthermore, these design rules are being found to be inadequate for the design of the longer longwall faces that are becoming increasingly common.

As mentioned previously, some longwall systems use twospeed pulse-amplitude-modulation (PAM)-type induction motors to try to alleviate some of the AFC problems. While this may give better starting torque, it does not address the mismatch problem and its effects on startup and steady-state performance.

\section{Application of Modern Drive Technology}

The technology of variable-speed drives (VSD's) has developed significantly in the last ten years. In the early 1980's, power-electronics-based VSD's did not have a good reputation in industry, mainly due to their poor reliability. The coal mining industry also reflected this general mood and, to a large degree, still does.

Modern VSD's are a combination of computer and power electronic technologies. They can now be built with high reliability, and the use of computers to control them means that sophisticated control algorithms can be implemented. These developments are relevant to the longwall coal mining industry, as inverter-controlled electric machines are capable of overcoming most, if not all, of the AFC problems cited above.

The potential advantages of using VSD's for the AFC motors are as follows.

1) The voltage drop problem is largely solved, because a VSD starts with a low input frequency and, hence, the slip is small. If the motor is being voltage fed, this will make the input current substantially lower than those obtained with mains frequency DOL starting. Furthermore, dependent on the control algorithm used, the input current to the machine is often a controlled quantity.

2) Because a VSD is a controlled voltage source, it is capable of compensating for voltage drops, especially under start and low-speed conditions. In addition to this, differential voltage drops in dual-drive systems can be compensated for. 
3) High starting torques, without oscillations or overcurrents, can be obtained.

4) Because of the smooth torques developed and variablespeed operation, the fluid coupling can be eliminated from the drive system, without any detrimental effects on starting performance or the gearboxes.

5) In dual-drive systems, coordinated control systems can be developed that enable correct load sharing, even with the mismatches in the other drive train components.

6) It allows the possibility of supersynchronous operation of the AFC motors.

7) It allows the possibility of slow-speed operation in light load conditions. This can lower energy costs and wear and tear on the AFC.

8) The tendency toward higher face voltages may not be necessary, since the voltage drop problems with inverter-fed machines are substantially less than DOLstart machines.

The disadvantages of using VSD's are as follows.

1) They probably would have a higher initial capital cost.

2) Motors would have to be overrated, due to the inverterdriven supply.

Remark 5: The development of a new generation of highvoltage Insulated gate bipolar transistors (IGBT's) makes it possible to build single-level IGBT-based inverters to supply the $1.1-\mathrm{kV}-\mathrm{rms}$ machines that are commonly used in mining applications in Australia. This technology makes inverters even more attractive, as they are simpler, have better performance, and will be of lower cost compared to gateturnoff-thyristor (GTO)-based or multilevel inverters that were traditionally required at these voltage levels.

If the advantages listed above are realized, then the possible higher capital cost of the drive system would be more than offset by the increased system up time. This increased up time would result from lower drive system component failure (since motors, fluid couplings, and gearboxes are not being overstressed) and far fewer AFC stalling incidents.

\section{CONCLUSION}

This paper has outlined the current difficulties experienced with longwall AFC drive systems. If the lengths of AFC's are to be increased, then these problems have to be addressed, in order to make the systems economical. Existing AFC design and control strategies have been shown to be inadequate for this task. The mining industry to date has been reluctant to accept en masse new technologies that hold the promise of solving virtually all the current problems of AFC systems. Power-electronic-driven AFC motors have been proposed as a solution to these problems, and the advantages and disadvantages were outlined.

The details of the implementation of an AFC drive system control strategy have been left to a companion paper [4].

\section{REFERENCES}

[1] L. A. Morley, J. L. Kohler, and H. M. Smolnikar, "A model for predicting motor load for an armored face-conveyor drive," IEEE Trans. Ind. Applicat., vol. 24, pp. 649-659, July/Aug. 1988.

[2] A. R. Broadfoot and R. E. Betz, "Prediction of the power requirements for a longwall armored face conveyor," IEEE Trans. Ind. Applicat., vol. 33, pp. 80-89, Jan./Feb. 1997.

[3] "The assessment of heavy duty conveyors in relation to modern mining systems, mining research and development establishment," National Coal Board, Burton-on-Trent, U.K., 1980.

[4] A. R. Broadfoot and R. E. Betz, "New control strategies for longwall armored face conveyors," this issue, pp. 387-394.

[5] D. O'Kelly and S. Simmons, Introduction to Generalized Electrical Machine Theory. New York: McGraw-Hill, 1968.

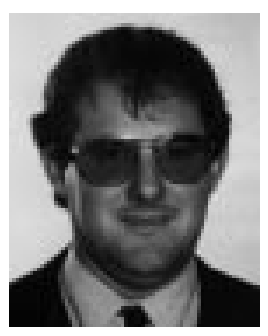

Alan R. Broadfoot (S'81-M'83) received the B.Eng. (Hon.), M.E., and Ph.D. degrees from the University of Newcastle, Newcastle, Australia, in 1981, 1985, and 1997, respectively.

From 1976 to 1980, he was a Trainee Engineer with Elcom Colleries Pty. Ltd., New South Wales, Australia. From 1981 to 1991, he served in various capacities within the company, including Group Project Engineer and Mine Electrical Engineer. In 1991, he joined AMP Control SWG Pty. Ltd., Newcastle, Australia, as Chief Engineer, and is currently General Manager of the Mining and Mineral Processing/Refining Business Unit.

Mr. Broadfoot is a past President of the Institution of Mining Engineers and a member of the Mining Industry Committee of the IEEE Industry Applications Society.

Robert E. Betz (M'92), for a photograph and biography, see this issue, p. 354. 